\title{
Low organisational justice and heavy drinking: a prospective cohort study
}

Running title: Low organisational justice and heavy drinking

\section{OEM/2007/032755; Final}

Anne Kouvonen, PhD, ${ }^{a}$ Mika Kivimäki, PhD, Prof., ${ }^{b, c}$ Marko Elovainio, PhD, ${ }^{d}$ Ari Väänänen, PhD, ${ }^{\mathrm{c}}$ Roberto De Vogli PhD, ${ }^{\mathrm{b}}$ Tarja Heponiemi, PhD,${ }^{\mathrm{d}}$ Anne Linna, MSc, ${ }^{c}$ Jaana Pentti, MSc ${ }^{c}$ and Jussi Vahtera, MD, PhD, Research Prof., ${ }^{c}$

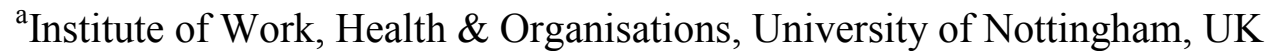

${ }^{b}$ Department of Epidemiology and Public Health, University College London, UK

${ }^{\mathrm{c}}$ Finnish Institute of Occupational Health, Helsinki, Finland

${ }^{\mathrm{d}}$ National Research and Development Centre for Welfare and Health (STAKES), Helsinki, Finland

Correspondence to:

Dr Anne Kouvonen, Institute of Work, Health \& Organisations, University of

Nottingham, 8 William Lee Buildings, Nottingham Science and Technology Park, University Boulevard, Nottingham NG7 2RQ, UK;

anne.kouvonen@nottingham.ac.uk

Word count: 203 (abstract);4380 (text)

Keywords: alcohol consumption; heavy drinking; organisational justice; psychosocial factors

Abbreviations: CI, confidence interval; ERI, effort-reward imbalance; OR, odds ratio; SD, standard deviation; SE, standard error; SES, socio-economic status.

\section{MAIN MESSAGES}

- Organisational justice is associated with employee health, but mechanisms underlying this association remain unclear.

- There was a weak association between low procedural justice at baseline and an increased likelihood of heavy drinking at follow-up in a large sample of Finnish public sector employees.

\section{POLICY IMPLICATIONS}

- Unfair managerial procedures might slightly increase risk of heavy drinking among employees. 


\section{ABSTRACT}

Objectives: To investigate whether low perceived organisational injustice predicts heavy drinking among employees.

Methods: Data from the prospective occupational cohort study, the 10-Town Study, related to 15290 Finnish public sector local government employees nested in 2432 work units, were used. Non-drinkers were excluded. Procedural, interactional and total organisational justice, heavy drinking ( $>=210 \mathrm{~g}$ of absolute alcohol per week) and other psychosocial factors were determined by means of questionnaire in 20002001 (phase 1) and 2004 (phase 2). Multilevel logistic regression analyses taking into account for the hierarchical structure of the data were conducted and adjustments were made for sex, age, socio-economic position, marital status, baseline heavy drinking, psychological distress and other psychosocial risk factors such as job strain and effort/reward imbalance.

Results: After adjustments, participants who reported low procedural justice at phase 1 were about 1.2 times more likely to be heavy drinkers at phase 2 compared with their counterparts with high justice. Low perceived justice in interpersonal treatment and low perceived total organisational justice were associated with an elevated prevalence of heavy drinking only in the socio-demographics adjusted model.

Conclusions: This is the first longitudinal study to show that low procedural justice is weakly associated with an increased likelihood of heavy drinking.

An increasing number of empirical studies suggest that the extent to which employees are treated with justice at workplace may affect their health. Organisational justice involves a procedural component and an interactional component. The procedural justice indicates whether decision-making procedures are consistently applied, suppress bias, and are accurate, correctable, and ethical.[1] The interactional component of organisational justice indicates whether people believe that their supervisor considers their viewpoints, shares information concerning decisionmaking, and treats individuals fairly and in a truthful manner.[2]

The health effects of organisational justice have been studied in several studies relying on large well-established British and Finnish employee cohorts. Lower level of organisational justice has been associated with increased mental health problems, higher medically certified sickness absence, incident coronary heart disease and higher self-reported morbidity, irrespective of other work-related psychosocial factors.[3][4][5][6][7][8][9][10] The mechanisms underlying these associations are not well known, although evidence has begun to emerge. In principle, organisational justice, like other psychosocial factors, could be related to ill health directly through physiological stress mechanisms and indirectly through behavioural risk factors, such as heavy drinking.[11]. According to Elovainio et al.,[12] low justice was related to increased low-frequency band systolic arterial pressure variability and reduced highfrequency heart rate variability, suggesting that cardiac dysregulation is one of the stress mechanisms through which a low perceived justice increases the risk of health problems. 
Evidence of the relationship between organisational justice and behavioural risk factors is scarce. Low procedural justice was cross-sectionally associated with high alcohol consumption in male hospital employees.[3] However, the temporal aspects of this association remained unclear and it is not known whether the association between low justice and heavy drinking was independent of other psychosocial work factors such as job strain,[13][14] or effort/reward imbalance (ERI),[14][15] which may also increase alcohol consumption.

Both procedural and interactional justice deal with how supervisors relate to their employees. This relationship may be crucial for mental health.[4] Research on depression suggests that life events in combination with humiliation and devaluation are more likely to lead to depression than life events alone.[16] Humiliation of employees may well be a component of low interactional justice. It is also possible that poor relations between supervisors and employees are a result of the supervisors being treated badly themselves by their superiors. Such a hierarchy of poor relations may be a reflection of a malfunctioning organisation in which low procedural justice may be either a cause or a consequence. Low procedural component also refers to a failure of the procedures used at the workplace to create clarity and consistency for the employees around decisions at work.[17]

Procedural justice can be related to heavy drinking through depression and other mental health problems, but evidence supporting this is indirect. Procedural justice predicted doctor-diagnosed depression in a longitudinal data[4] and it has also been linked with minor psychiatric morbidity both cross-sectionally[7] and longitudinally.[4] In the Whitehall II study, a favourable change in justice reduced the immediate risk of psychiatric morbidity, whilst an adverse change increased the immediate and longer-term risk.[10] Mental health problems, depression in particular, can increase substance use.[18][19]

Data from the 10-Town Study, an ongoing large-scale prospective occupational cohort study, have enabled our examination of the longitudinal association between perceived organisational justice and heavy drinking. The purpose of our study was to examine whether the levels of perceived procedural, interactional and total organisational justice (a combined index of procedural and interactional justice) were associated with subsequent heavy drinking independently of other psychosocial characteristics of the work environment. We used multilevel modelling to take into account the fact that individual employees are nested in social units comprised of workplaces.[20]

\section{METHODS}

\section{Study design and study population}

The ongoing prospective Finnish 10-Town cohort study focuses on the entire personnel of ten local governments. The baseline data on organisational justice, alcohol consumption, and other factors were collected in 2000 and 2001 through selfadministered questionnaires from 32299 women and men. The response rate was $67 \%$. Of these employees, 24196 were still contracted to the towns at the time of the follow-up survey in 2004. The response rate at phase 2 for individuals who remained 
employed at the target organisations at phase 2 survey and who had responded at phase 1 was $79 \%(n=19077)$. The average follow-up was 3.6 years.

This study focused on those 15290 participants (11 745 women and 3545 men) with data on organisational justice at baseline, who reported alcohol use at follow-up, who worked in work units composed of at least three employees, and who had no missing values for any of the covariates. Non-drinkers $(n=2437)$ had been excluded, because they represent two very different populations; teetotallers and ex-drinkers. Nondrinkers can have strong special reasons for abstaining. Ex-drinkers include recovered alcoholics and people with diseases that are worsened by drinking, whereas teetotallers may have health problems, religious or moral reasons for abstaining.[21]

The final dataset included individuals (employees) nested within 2432 work units in 10 towns. The work unit of each respondent was identified from the employers' records based on a five-level organisational hierarchy classification. Work unit was the lowest level in the organisational hierarchy.

Any differences with the eligible population were small. The figures for the final cohort (baseline) compared with the eligible population $(n=47351)$ were as follows: mean age 44.8 compared with 44.5 years and the proportion of women $77 \%$ compared with $72 \%$.

Approval from the ethics committee of the Finnish Institute of Occupational Health was obtained for the study.

\section{Assessment of organisational justice}

Organisational justice was measured using the scale developed by Moorman.[2] The procedural justice scale (seven items, Cronbach's alpha $=0.91$ at baseline; 4-year testretest correlation coefficient $r=0.53$ ) measured the degree to which respondents considered the procedures used at the workplace to be designed to collect accurate information necessary for making decisions, to provide opportunities to appeal or challenge the decision, to generate standards so that decisions could be made with consistency, and to hear the concerns of all those affected by the decision.

The interactional justice scale[2] (six items, Cronbach's alpha $=0.92$ at baseline; 4year test-retest correlation coefficient $r=0.44$ ) measured whether respondents thought that their supervisors were able to suppress personal biases, to treat subordinates with kindness and consideration, and to take steps to deal with subordinates in a truthful manner.

In both scales responses were given on a five-point scale ranging from 1 = "strongly disagree" to 5 = "strongly agree".

In addition to the scales of procedural and interactional organisational justice, we constructed a scale of total organisational justice by calculating a mean score of these two scales (13 items, Cronbach's alpha $=0.92$ at baseline; 4 -year test-retest correlation coefficient $r=0.53$ ). The correlation between the two sub-scales was $0.49(\mathrm{p}<.001)$. The intra class correlation (ICC) of the total justice was $15 \%$. ICC evaluates between-group variance relative to total (between and within) variance.[22] 
The within-group (interrater) agreement index $\left(\mathrm{r}_{\mathrm{wg}}\right)$ can be used to measure the agreement between workers in the same work unit regarding their perception of organisational justice. An $r_{w g}$ index value of 0.70 or higher is perceived as an acceptable agreement.[23] In our sample, the average deviation of an individual's perception of total organisational justice from the mean level of her/his work unit was 0.89 , indicating a significant homogeneity in the perceptions of organisational justice within a work unit.

The participants were divided into 3 groups based on the distribution of the organisational justice scores. The bottom third indicated a low level of justice, the middle third a medium level, and the top third a high level of justice.

\section{Assessment of heavy drinking}

Heavy drinking was assessed at baseline and follow-up. The questions of drinking habits were framed as follows: "Have you ever consumed at least one glass of some alcoholic drink?" "How much you consume the following alcoholic drinks on average (beer per week; wine or other mild drinks per week; spirits per month). The habitual frequency and amount of beer, wine and spirits intake was transformed into grams of absolute alcohol per week.[24] One unit of pure alcohol $(12 \mathrm{~g})$ is equal to a 12-cl glass of wine, a single 4-cl measure of spirits, or a 33-cl bottle of beer. A dichotomous variable was created to represent heavy drinking, with a cutoff point corresponding to the weekly consumption of $210 \mathrm{~g}$ or more absolute alcohol.[25] All other respondents reporting alcohol intake below these limits (light-moderate drinkers) were placed in the reference category.

\section{Assessment of covariates at baseline}

The demographic baseline characteristics used in the analysis included sex, age, socioeconomic status (SES) based on ISCO-88 classification (managers and professionals, associate professionals, clerks, service workers, and manual workers), and marital status (married or co-habiting vs. single, divorced, or widowed). Sex, age and occupational status were obtained from the employers' registers and age was grouped into three categories (19-34; 35-50; 51-62 years).

Psychological distress was measured by the 12-item version of the General Health Questionnaire (Cronbach's alpha $=0.89$ ). [26] Individuals scoring $>=4$ were estimated to have high mental distress.

Assessment of job strain was based on a modified Job Content Questionnaire[27] comprised of the job demand scale ( 3 items, Cronbach's alpha $=0.75$ at baseline) and job control scale ( 9 items, Cronbach's alpha $=0.82$ at baseline). The responses were given on a Likert scale of $1=$ "very little" to $5=$ "very much". The total scores for each of the two constructs were computed. To construct the job strain measure, the means of job demand scores were subtracted from the means of job control scores.[6][28][29][30] All participants were divided into 3 groups based on the distribution of this score.

Effort in work was measured with the following question: "How much do you feel you invest in your job in terms of skill and energy?" Rewards were assessed with a scale containing three questions about feelings of getting in return from work in terms 
of (1) income and job benefits, (2) recognition and prestige, and (3) personal satisfaction (Cronbach's alpha $=0.63$ at baseline).[31] Response format for all the questions was a five-point Likert scale ranging from $1=$ "very little" to $5=$ "very much". The indicator of ERI was obtained by calculating the ratio between the response score in the effort scale and the mean response score in the reward scale. The resulting quotient was divided into thirds to indicate low, medium, and high ERI.

\section{Statistical analysis}

Because the participants were nested within work units, we applied multilevel logistic regression analysis method to study the associations between perceived organisational justice at baseline and heavy drinking at follow-up among current drinkers. This method accounts for the non-independence of observations within groups. The results of the modelling are presented as odds ratios (ORs) and their 95\% confidence intervals (CIs). The hypothetically most favourable condition (highest level of justice) was selected as a reference category in each indicator of perceived organisational justice. The analysis was conducted in three steps. In the first model, only sex, age, SES and marital status were controlled for. The second model also included baseline heavy drinking and psychological distress. The third model was additionally adjusted for job strain and ERI.

The statistical significance of interactions between justice variables and sex were tested by including interaction terms.

All models were fitted using the SAS version 9.1.3 program package (SAS Institute, Cary, NC, USA). The multilevel analyses were performed by using the Glimmix procedure.

\section{RESULTS}

The baseline study characteristics are shown in table 1 . The majority of the participants were 35 to 50 -years old and $76.9 \%$ were married. The most common SES group was managers and professionals $(36.2 \%)$, followed by service workers $(23.4 \%)$.

In the follow-up, $11.9 \%$ of the participants were heavy drinkers who consumed $210 \mathrm{~g}$ or more of absolute alcohol a week. Heavy drinking was more frequent among men (25.4\%) than among women (7.9\%). The percent of heavy drinkers was higher in the two oldest than in the youngest age group. Moreover, heavy drinking was more frequent among managers and professionals (14.1\%) as well as among manual workers $(15.9 \%)$ than other SES groups. ( $p$ value for differences in all cases $<0.001$ except with age where $\mathrm{p}=0.011$; Data not shown.)

The level of perceived organisational justice in the final cohort corresponded well with that among the respondents of the first survey. The mean scores for total organisational justice were $3.38(\mathrm{SD}=0.77$, range $1-5)$ for the first and $3.38(\mathrm{SD}=$ 0.79 , range 1-5) for the latter group. Therefore bias due to selection procedures or sample attrition seems unlikely.

Women reported slightly more often high interactional justice than men. Workers in the oldest and the youngest age groups experienced higher levels of procedural and 
total justice more often than the workers in the middle age group. High justice was more frequent in managers and professionals as well as in service workers than in other SES groups. As expected, high job strain and high ERI were connected with low organisational justice. ( $p$ value for difference $<0.001$ in all cases in Chi Square 2tailed test) (data not shown).

Only $4.4 \%$ of the participants were exposed to high procedural but low interactional justice, whereas $6.6 \%$ were exposed to high interactional but low procedural justice. $19.4 \%$ of the participants were exposed to high procedural and high interactional justice.

There were no significant differences in the means of perceived procedural, interactional and total justice between the baseline and follow-up surveys. At baseline the means (SD) of procedural, interactional and total organisational justice were 3.15 (0.86), $3.64(0.92)$ and $3.38(0.77)$, respectively. In the follow-up survey the corresponding figures were $3.11(0.88), 3.66(0.93)$ and $3.37(0.79)$, respectively $(\mathrm{p}<$ $0.001)$. Range was $1-5$ in all cases.

734 participants were drinkers at baseline but non-drinkers at follow-up. 687 participants were heavy drinkers at baseline but non-heavy drinkers at follow-up. The mean of perceived total organisational justice in participants who were drinkers at baseline but teetotallers at follow-up was 3.45 ( $\mathrm{SD}=0.78$; range 1.08-5.00).

There were differences in heavy drinking between work units. In the multilevel model, the component variance of work unit was significant (Var $=0.039, \mathrm{SE}=0.005$, $\mathrm{p}<.001$ ). In $48 \%$ of the work units there were no heavy drinkers while in $10 \%$ of work unit at least a third of the participants were heavy drinkers. 
Table 1 Baseline characteristics of participants $(n=15083)$

(Figures in parentheses are percentages.)

Characteristics No of participants

All

15290

Sex

Women

Men

$11745(76.8)$

$3545(23.2)$

Age (in years)

19-34

$1797(11.8)$

35-50

$9124(59.7)$

$51-62$

$4369(28.6)$

Socio-economic

status

Managers and

$5536(36.2)$

professionals

Associate

$2246(14.7)$

professionals

Clerks

$1532(10.0)$

Service workers

$3570(23.4)$

Manual workers

$2401(15.7)$

Marital status

Married/

cohabiting

Other

$11766(76.9)$

$3524(23.1)$

Psychological

distress

No

$11311(74.0)$

Yes

$3979(26.0)$

Job strain

Low

Medium

$5072(33.1)$

$5319(34.8)$

High $\quad 4899(32.0)$

Effort/reward

imbalance

Low

$4682(30.6)$

Medium

$5795(37.9)$

High

$4813(31.5)$ 
Table 2 shows the associations between perceived organisational justice variables at baseline and heavy drinking at follow-up. After adjustment for sex, age, SES, marital status, heavy drinking at baseline, psychological distress, job strain and ERI, low perceived procedural justice was associated with approximately $16 \%$ higher odds of heavy drinking. Low perceived interactional justice $(\mathrm{OR}=1.19,95 \% \mathrm{CI}$ : 1.05 to $1.34)$ and low perceived total justice (OR $=1.23,95 \% \mathrm{CI}$ : 1.09 to 1.40$)$ were associated with a higher likelihood of heavy drinking in the model adjusted for sociodemographics, but attenuated to non-significant after further adjustments.

We conducted the analyses also using the higher $(250 \mathrm{~g})$ and lower $(190 \mathrm{~g})$ cut-off points of heavy drinking.[14] The results were to the similar direction as with the chosen cut-off point. In the fully adjusted model, the OR for heavy drinking in the lowest tertile of perceived procedural justice was 1.15 (95\% CI: 0.93 to 1.41 ) with the cut-off point of $250 \mathrm{~g}$ and 1.21 (95\% CI: 1.05 to 1.38 ) with the cut-off point of $190 \mathrm{~g}$. (Data not shown.)

We also conducted SES-stratified analyses for perceived procedural justice. The results were into the same direction in all SES groups, although low perceived procedural justice was significantly associated with a higher likelihood of heavy drinking only among managers and professionals ( $\mathrm{OR}=1.28,95 \% \mathrm{CI}: 1.01$ to 1.62$)$. (Data not shown.)

The effect of an interaction between each indicator of perceived organisational justice and sex on heavy drinking at follow-up was tested by including the corresponding interaction term in regression models that already included the main effects. No sex interactions were found ( $p$ values for interaction: procedural justice $p=0.184$, interactional justice $p=0.802$ and total justice $p=0.772$ ).

To test the possibility of reversed causality, we examined whether baseline heavy drinking predicted perceived procedural justice at follow-up after adjustment for sex, age and baseline procedural justice. We did not found significant association. Thus, reverse causation is unlikely to explain the observed association between perceived procedural justice and subsequent heavy drinking. (Data not shown.) 
Table 2 Associations of perceived procedural, interactional and total organisational justice at baseline with heavy drinking at follow-up: adjusted odds ratios and their 95 percent confidence intervals (95\% Cls) from multilevel logistic regression models $(n=15290)$.

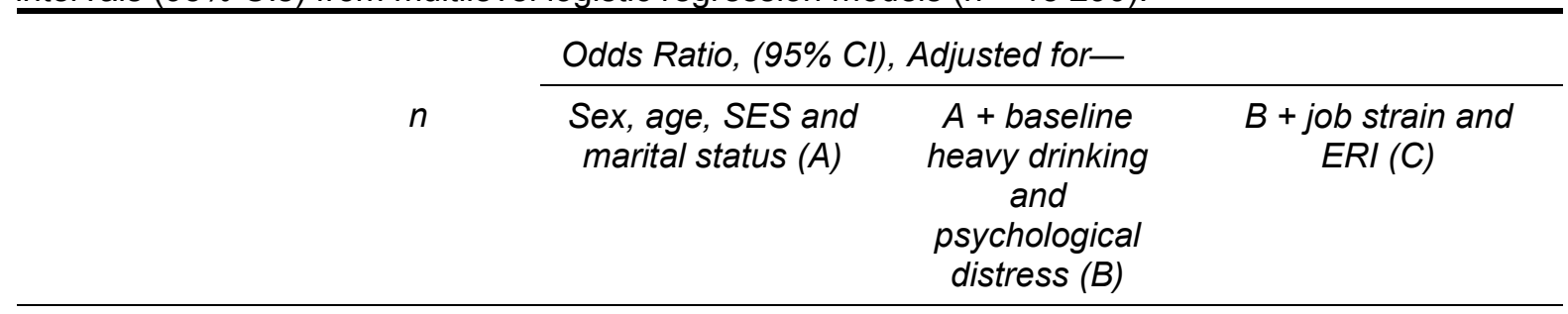

15290

\section{Procedural justice}

High

Medium

Low
5191

4713

5386
1

1.04 (0.91 to 1.18$)$

1.23 (1.09 to 1.39$)$
1

1.07 (0.92 to 1.24$)$

$1.13(0.97$ to 1.30$)$
1

1.08 (0.93 to 1.26$)$

$1.16(1.00$ to 1.35$)$

\section{Interactional \\ justice}

\begin{tabular}{lcccc} 
High & 5457 & 1 & 1 & 1 \\
$\begin{array}{l}\text { Medium } \\
\text { Low }\end{array}$ & 4562 & $1.04(0.91$ to 1.18$)$ & $1.01(0.87$ to 1.17$)$ & $1.02(0.88$ to 1.19$)$ \\
justice & 5271 & $1.19(1.05$ to 1.34$)$ & $1.05(0.91$ to 1.22$)$ & $1.08(0.93$ to 1.25$)$ \\
High & & & & \\
Medium & 5349 & 1 & 1 & 1 \\
Low & 4749 & $1.11(0.98$ to 1.26$)$ & $1.08(0.93$ to 1.26$)$ & $1.10(0.95$ to 1.28$)$ \\
& 5192 & $1.23(1.09$ to 1.40$)$ & $1.07(0.93$ to 1.24$)$ & $1.10(0.95$ to 1.29$)$ \\
\hline
\end{tabular}

ERI, effort/reward imbalance; SES, socio-economic status.

\section{DISCUSSION}

Research on organisational justice provides insight into an often forgotten aspect of the impact of work organisation on health. So far, only a few studies have examined the association between organisational justice and health-risk behaviours. This prospective study showed that low levels of perceived procedural organisational justice predicted heavy drinking in women and men in a large well characterised cohort of Finnish local government employees. The associations were not accounted for by baseline characteristics such as sex, age, marital status, socio-economic position, baseline heavy drinking, psychological distress, job strain and effort/reward imbalance. The odds ratios were rather small, but still potentially important from a public health point of view as heavy alcohol consumption appears to increase morbidity and mortality risk, whereas moderate alcohol consumption has been shown to be associated with a decreased risk.[32][33][34]

Conditions of low control and low organisational justice can occur simultaneously in the same work environment.[35] This was also the case in the present study, as $45.8 \%$ of participants with high job strain and $47.2 \%$ of participants with high ERI also were in the lowest tertile of procedural justice ( $<<0.001$ in both cases). However, 
employees who have control over their jobs may still experience that their supervisor is unreliable, inconsiderate and untruthful. Moreover, favourable procedural justice evaluation may act as a mediating mechanism through which a high degree of job control protects employees against the development of strain symptoms.[35]

In our study heavy drinking was predicted by the procedural rather than the interactional component of perceived justice. This is in accordance with earlier evidence on other health outcomes[3][7]; previous research reports less consistent health effects for interactional justice than for procedural justice. This suggests that a low justice work environment characterised by unjust organisational policies, practices, and procedures is a greater risk to health and health behaviours than unfair treatment from an immediate supervisor.

Our findings suggest that the concept of organisational justice adds to prior research based on the established theoretical models of psychosocial risk factors. This view is also supported by studies on other outcomes, such as psychiatric disorders,[4][10] self-rated health,[5] coronary heart disease,[6] cardiac dysregulation,[9] and smoking.[36]

Earlier evidence on the association between psychosocial work-related factors and alcohol use is mixed, mostly cross-sectional, and mostly relates to the job strain model. The job strain model postulates that job strain emerges from the combination of high job demands and low job control generating increased risk of ill health and adverse health behaviours.[37] Some studies have found job strain variables (high job strain, high demands, low control or passive jobs) to be associated with alcohol consumption, [38] alcohol abuse-dependence,[39] or alcohol use.[13] However, these findings are contradicted by several studies in which either no association was found between alcohol use and job strain variables,[40][41][42][43] or the directionality of the associations was partly contrary to what was expected.[14] When taking into account also other work stressors than job strain, research in general supports a weak relation of work-related stressors to elevated alcohol consumption and problem drinking.[44][45]

A more recent model of occupational stress, the ERI model, is based on the assumption that the mismatch between high effort spent and low rewards (financial, self-esteem, and social) gained from work, can result in an increased risk of ill health.[46] The evidence of the association between ERI and alcohol use is limited, but the results from the Whitehall II cohort indicate an increased risk of alcohol dependence in men with high ERI.[15] Low effort in both women and men and low rewards in men have also been associated with heavy drinking.[14]

The primary focus of the job strain model is on task level characteristics. [5] It is possible that organisational justice captures more basic elements of social structure. Moreover, unlike the models of job strain and ERI, justice approach is directly focused on managerial treatment and managerial procedures. Therefore, it covers all kind of unfairness, not only that arising from disproportionate demands in relation to job control[6] or the specific exchange process between efforts and rewards. Employees may be affected not only by rewards as such but also by procedures used to determine how these rewards will be distributed.[7] 
Low organisational justice has been associated with occupational strain[34] and to negative emotional reactions.[47] Both of these have been associated with unhealthy patterns of cardiovascular and immunological response as well as with health problems.[48] The workplace stress paradigm suggests that employee alcohol use may represent a strategy to cope with negative emotions resulting from exposure to adverse work conditions. [44] Individuals have been suggested to use alcohol to reduce negative mood states.[49] Therefore, low organisational justice can represent a source of stress and negative emotional reactions at work that can potentially contribute to health problems[34] and health-risk behaviours such as heavy drinking. In their longitudinal study, Elovainio et al. [8] found that the effect of low organisational justice on minor psychiatric morbidity, such as depression and selfreported health, was mediated by sleeping problems. Both depression and sleeping problems have been related to elevated alcohol consumption in previous studies.[50][51][52][53]

However, the observed association between low organisational and heavy drinking was weak. This is in line with Frone's[44] suggestion that although most adults consume alcohol, it is unlike that most workers use alcohol to cope with adverse work conditions. Many other coping mechanisms relieve the negative emotions resulting from work stressors more effectively and have fewer negative side effects. On the other hand, people have numerous non-work-related reasons for their heavy drinking. The features of procedural justice may also reflect social integration and social support.[35]

Even if strictly speaking it is unknown where the heavy drinking behaviour occurs, our measure assessed most likely heavy drinking out of work. A recent national survey in the US showed that an estimated that only $7 \%$ of the workers use alcohol during the workday.[54] If our results reflect a causal association and the impact that the work environment may have in out-of-work behaviours, this is of practical importance.

\section{Study strengths and limitations}

Our evidence was based on a well-characterised cohort, prospective design and repeated measurements. The response rate was satisfactory and the data were fairly representative including both manual and non-manual occupations. Large sample size allowed detection of weak effects. Moreover, multilevel modelling was used and we adjusted for multiple covariates thereby minimising confounding bias. Although the possibility of confounding by an unknown factor can never be excluded, a major bias in our study is unlikely. The contribution of procedural justice to heavy drinking largely persisted after control for socio-demographic factors, minor psychological distess and psychosocial factors. The longitudinal design allowed us to address the issue of reverse causality. No evidence was found to support the hypothesis that a perception of low procedural justice would represent a consequence of being a heavy drinker.

However, interpretations of these findings should take into consideration study limitations. First, this study assessed heavy drinking and organisational justice with self-reports. It is well know that self-report data on alcohol use can be subject to underreporting.[55] This can lead to an underestimation of the real association 
between organisational justice and heavy drinking. Moreover, underreporting of alcohol use may be associated with organisational justice. If exposure to low organisational justice at the baseline increases underreporting of alcohol use more at the baseline than at the follow-up, it might lead to an apparent increase of drinking among those exposed to low organisational justice and inflate the association between organisational justice and subsequent alcohol use.

As justice was self-reported, the assessment may reflect both the respondent and the work environment. The individual perception is assumed to be essential for organisational justice to affect health.[20] However, previous studies have suggested that self-reported justice levels may accurately reflect organisational reality[56][57] and both individual-level justice scores and more objective ecological scores have been shown to be predictive of mental health. [8] Indeed, people may also take into account the experiences of others when they form justice judgements.[58]

Second, our alcohol consumption measure did not differentiate between escapist and social drinking. Heavy drinking can be problematic but it may as well reflect a more socially oriented non-escapist and necessarily not problematic type of behaviour.[59]

Third, we cannot rule out the possibility that heavy drinking and low perceived organisational justice could both be products of an unmeasured third factor. For example, personality characteristics such as hostility as well as other factors such as mood, depression and non-work related risks may affect both the perception of justice and heavy drinking. However, previous studies on health-related personality traits, such as hostility and anxiety, do not indicate a strong relationship between personality and justice perceptions.[60]

Fourth, the response rates of our surveys were satisfactory but attrition was slightly higher among heavy drinkers. Such selection may have caused a healthy worker effect and thus attenuated the association between organisational justice and heavy drinking.

Fifth, the standard measure of ERI was not available in this study. However, both studies using original and proxy measures have found support for the ERI model regardless of the measure.[61] Nevertheless, there is a possibility that our crude measure did not fully capture the ERI model and also the Cronbach alpha for the rewards scale was rather moderate.

Sixth, we could not assess the effect of low organisational justice exposure duration, since the length of the period in which the worker had been exposed to low organisational justice by baseline was unknown to us.

Finally, as our evidence was based on female-dominated cohort of public sector workers, further research is needed on other occupational groups and in other countries. Further research is also needed to directly investigate the mechanisms that may underlie the effects of low procedural justice on health-risk behaviours such as heavy drinking. 


\section{CONCLUSIONS}

In this study, we found a $16 \%$ excess likelihood of heavy drinking in employees with low procedural justice perceptions after four years of follow up. Our results on heavy drinking offer further support for the previously reported findings that low organisational justice can pose health risk for employees not attributable to other work-related psychosocial factors. 


\section{ACKNOWLEDGEMENTS}

The work presented in this paper was supported by grants from the Academy of Finland (projects 105195, 110451 and 117604), the Finnish Work Environment Fund (project 103432) and the participating towns.

Competing interest statement: No competing interests.

Copyright statement: "The Corresponding Author has the right to grant on behalf of all authors and does grant on behalf of all authors, an exclusive licence (or non exclusive for government employees) on a worldwide basis to the BMJ Publishing Group Ltd to permit this article (if accepted) to be published in OEM and any other BMJPGL products and sublicences such use and exploit all subsidiary rights, as set out in your licence (http://oem.bmjjournals.com/misc/ifora/licenceform.shtml)." 


\section{REFERENCES}

1 Leventhal GS. What should be done with equity theory? New approaches to the study of fairness in social relationships. In: Gergen KS, Greenberg MS, Willis RH, editors. Social exchange: Advances in theory and research. New York: Plenum, 1980. p. 27-55.

2 Moorman RH. Relationship between organizational justice and organizational citizenship behaviors: Do fairness perception influence employee citizenship? J Appl Psychol 1991;76:845-55.

3 Kivimäki M, Elovainio M, Vahtera J, et al. Organisational justice and health of employees: prospective cohort study. Occup Environ Med 2003;60:27-34.

4 Kivimäki M, Elovainio M, Vahtera J, et al. Association between organisational inequity and incidence of psychiatric disorders in female employees. Psychol Med 2003;33:319-26.

5 Kivimäki M, Ferrie JE, Head J, et al. Organisational justice and change in justice as predictors of employee health: the Whitehall II study. J Epidemiol Community Health 2004;58:931-7.

6 Kivimäki M, Ferrie JE, Brunner E, et al. Justice at work and reduced risk of coronary heart disease among employees. The Whitehall II Study. Arch Intern Med 2005; 165:2245-51.

7 Elovainio M, Kivimäki M, Vahtera J. Organizational justice: Evidence of a new psychosocial predictor of health. Am J Public Health 2002;92:105-8.

8 Elovainio M, Kivimäki M, Vahtera J, et al. Sleeping problems and health behaviors as mediators between organizational justice and health. Health Psychol 2003;22:28793.

9 Elovainio M, Kivimäki M, Puttonen S, et al. Organisational injustice and impaired cardiovascular regulation among female employees Occup Environ Med 2006;63:1414 .

10 Ferrie JE, Head J, Shipley MJ, et al. Injustice at work and incidence of psychiatric morbidity: the Whitehall II study. Occup Environ Med 2006;63:443-50.

11 Brunner E, Marmot M. Social organisation, stress, and health. In: Marmot M, Wilkinson RG, eds. Social determinants of health. Oxford: Oxford University Press, 1999:17-43.

12 Elovainio M, Leino-Arjas P, Vahtera J, et al. Justice at work and cardiovascular mortality: a prospective cohort study. J Psychosom Res 2006;6:271-4.

13 San Jose B, van de Mheen H, van Oers JAM, et al. Adverse working conditions and alcohol use in men and women. Alcohol Clin Exp Res 2000;24:1207-13. 
14 Kouvonen A, Kivimäki M, Cox SJ, et al. Job strain, effort-reward imbalance and heavy drinking: a study in 40,851 employees. J Occupat Environ Med;47:503-13.

15 Head J, Stansfeld SA, Siegrist J. The psychosocial work environment and alcohol dependence: a prospective study. Occup Environ Med 2004;61:219-44.

16 Brown GW, Harris TO, Hepworth C. Loss, humiliation and entrapment among women developing depression: a patient and non-patient comparison. Psychol Med 1995;25:7-21.

17 Theorell T. Commentary. Occup Environ Med 2003;60:33-4.

18 Lecrubier Y. Depressive illness and disability. European Neuropsychopharmacology 2000;10 (Suppl.):S439-S443.

19 Goldberg RJ, Steury S. Depression in the work place: costs and barriers to treatment. Psychiatric Services 2001;52:639-43.

20 Elovainio M, Kivimäki M, Steen N, et al. Job decision latitude, organizational justice and health: multilevel covariance structure analysis. Soc Sci Med 2004;58: 1659-69.

21 Eward AM, Wolfe R, Moll P, et al. Psychosocial and behavioral factors differentiating past drinkers and life-long abstainers. Am J Public Health 1986;76:6870 .

22 Merlo J, Chaix B, Yang M, et al. A brief conceptual tutorial of multilevel analysis in social epidemiology: linking the statistical concept of clustering to the idea of contextual phenomenon. J Epidemiol Community Health 2005;59:443-9.

23 James LR, Demaree RG, Wolf G. Estimating within-group interrater reliability with and without response bias. J Appl Psychol 1984;69:85-98.

24 Kaprio J, Romanov K, Sarna S, et al. Genetic influences on use and abuse of alcohol: a study of 5638 adult Finnish twin brothers. Alcohol Clin Exp Res 1987;11: 349-56.

25 Rimm EB, Williams P, Fosher K, et al. Moderate alcohol intake and lower risk of coronary heart disease: meta-analysis of effects on lipids and haemostatic factors. $B M J$ 1999;319:1523-8.

26 Goldberg D, Williams P. A user's guide to the General Health Questionnaire. Berkshire: NFER-Nelson Publishing Co., 1988. 
27 Karasek R. Job content questionnaire and user's guide. Revision 1.1. Los Angeles, CA: Department of Industrial and Systems Engineering, University of Southern Los Angeles, 1985.

28 Landsbergis PA, Schnall PL, Warren K, et al. Association between ambulatory blood pressure and alternative formulations of job strain. Scand $J$ Work Environ Health 1994;20:349-63.

29 Kivimäki M, Head J, Ferrie JE, et al. Work stress, weight gain and weight loss: Evidence for bidirectional effects of job strain on body mass index in the Whitehall II study. Int J Obesity 2006;30:982-7.

30 Kivimäki M, Head J, Ferrie JE, et al. Why is evidence on job strain and coronary heart disease mixed? An illustration of measurement challenges in the Whitehall II study. Psychosom Med 2006;68:398-401.

31 Taris TW, Kalimo R, Schaufeli WB. Inequity at work: its measurement and association with worker health. Work Stress 2002;16:287-301.

32 Vahtera J, Poikolainen K, Kivimäki M, et al. Alcohol intake and sickness absence: a curvilinear relation. Am J Epidemiol 2002;156:969-76.

33 Corraro G, Rubbiati L, Bagnardi V, et al. Alcohol and coronary heart disease: a meta-analysis. Addiction 2000;95:1505-23.

34 Poikolainen K. Alcohol and mortality: a review. J Clin Epidemiol 1995;48:45565.

35 Elovainio M, Kivimäki M, Helkama K. Organisational justice evaluations, job control, and occupational strain. J Appl Psychol 2001;86:418-24.

36 Kouvonen A, Vahtera J, Elovainio M, et al. Organisational justice and smoking: Finnish Public Sector Study. J Epidemiol Community Health 2007;61:427-33.

37 Karasek RA, Theorell T. Healthy work. Stress, productivity, and the reconstruction of working life. New York: Basic Books, 1990.

38 Mensch BS, Kandel DB. Do job conditions influence the use of drugs? J Health Soc Behav 1988;29:169-84.

39 Crum RM, Muntaner C, Eaton WW, et al. Occupational stress and the risk of alcohol abuse and dependence. Alcohol Clin Exp Res 1995;19:647-55. 
40 Alterman T, Shekelle RB, Vernon SW, et al. Decision latitude, psychologic demand, job strain, and coronary heart disease in the Western Electric Study. Am J Epidemiol 1994;139:620-7.

41 van Loon AJM, Tijhuis M, Surtees PG, et al. Lifestyle risk factors for cancer: the relationship with psychosocial work environment. Int J Epidemiol 2000;29:785-92.

42 Lallukka T, Sarlio-Lähteenkorva S, Roos E, et al. Working conditions and health behaviours among employed women and men: the Helsinki Health Study. Prev Med 2004;38:48-56.

43 Wiesner M, Windle M, Freeman A. Work stress, substance use, and depression among young adult workers: an examination of main and moderator effect model. J Occup Health Psychol 2005;10:83-96.

44 Frone MR. Work stress and alcohol use. Alcohol Res Health 1999;23:284-91.

45 Ragland DR, Greinet BA, Yen IH, et al. Occupational stress factors and alcoholrelated behavior in urban transit operators. Alcohol Clin Exp Res 2000;24:1011-9.

46 Siegrist J. Adverse health effects of high effort/low reward conditions. J Occup Health Psychol 1996;1:27-41.

47 Weiss HM, Suckow K, Cropanzano RS. Effects of justice conditions on discrete emotions. J Appl Psychol 1999;84:786-94.

48 Räikkönen K, Keltikangas-Järvinen L, Adlercreutz H, et al. Psychosocial stress and the insulin resistance syndrome. Metabolism 1996;45:1533-8.

49 Cooper ML, Frone MR, Russell M, et al. Drinking to regulate positive and negative emotions: a motivational model of alcohol use. J Pers Soc Psychol 1995; 69:990-1005.

50 Kivimäki M, Vahtera J, Pentti J, et al. Factors underlying the effect of organisational downsizing on health of employees: longitudinal cohort study. BMJ 2000;320:971-5.

51 Niedhammer I, Goldberg M, Leclerc A, et al. Psychosocial work environment and cardiovascular risk factors in an occupational cohort in France. J Epidemiol Community Health 1998;52:93-100.

52 Plant ML, Plant MA, Foster J. Stress, alcohol, tobacco and illicit drug use amongst nurses: a Scottish study. J Adv Nurs 1992;17:1057-67. 
53 Tachibana H, Izumi T, Honda S, et al. A study of the impact of occupational and domestic factors on insomnia among industrial workers of a manufacturing company in Japan. Occup Med (Lond) 1996;46:221-7.

54 Frone MR. Prevalence and distribution of alcohol use and impairment in the workplace: a U.S. national survey. J Stud Alcohol 2006;67:147-56.

55 Midanik LT. Validity of self-reported alcohol use: a literature review and assessment. Br J Addict 1988;83:1019-30.

56 Maurer TJ, Raju NS, Collins WC. Peer and subordinate performance appraisal measurement equivalence. $J$ Appl Psychol 1998;83:693-702.

57 Riggio RE, Cole EJ. Agreement between subordinate and superior ratings of supervisory performance and effects on self and job satisfaction. $J$ Occup Organisational Psychol 1992;65:151-8.

58 Lind EA, Kray L, Thompson L. The social construct of injustice: Fairness judgements in response to own and other's unfair treatment by authorities.

Organ Behav Hum Decis Process 1998;75:1-22.

59 Grunberg L, Moore S, Anderson-Connolly R, et al.. Work stress and self-reported alcohol use: the moderating role of escapist reasons for drinking. $J$ Occup Health Psychol 1999;4:29-36.

60 Elovainio M, Kivimäki M, Vahtera J, et al. Personality as a moderator in the relations between perceptions of organizational justice and sickness absence. $J$ Vocat Behav 2003;63:379-95.

61 van Vegchel N, de Jonge J, Bosma H, Schaufeli W. Reviewing the effort-reward imbalance model: drawing up the balance of 45 empirical studies.

Soc Sci Med 2005;60:1117-31. 